\title{
Eduard IRICINSCHI, Holger M. ZELLENTIN, (éds.), Heresy and Identity in Late Antiquity
}

Tübingen, Mohr Siebeck, 2008, 407 p.

\section{Anna Van den Kerchove}

\section{(2) OpenEdition} Journals

Édition électronique

URL : http://journals.openedition.org/assr/21587

DOI : $10.4000 /$ assr.21587

ISSN : 1777-5825

Éditeur

Éditions de l'EHESS

Édition imprimée

Date de publication : 31 décembre 2009

Pagination : $75-342$

ISBN : 978-2-7132-2218-4

ISSN : 0335-5985

Référence électronique

Anna Van den Kerchove, «Eduard IRIIINSCHI, Holger M. zelLentin, (éds.), Heresy and Identity in Late Antiquity ", Archives de sciences sociales des religions [En ligne], 148 | octobre-décembre 2009, document 148-69, mis en ligne le 27 janvier 2010, consulté le 21 septembre 2020. URL : http:// journals.openedition.org/assr/21587 ; DOI : https://doi.org/10.4000/assr.21587

Ce document a été généré automatiquement le 21 septembre 2020

(c) Archives de sciences sociales des religions 


\title{
Eduard IRICINSCHI, Holger M.
} ZELLENTIN, (éds.), Heresy and Identity in Late Antiquity

Tübingen, Mohr Siebeck, 2008, 407 p.

\author{
Anna Van den Kerchove
}

\section{RÉFÉRENCE}

Eduard IRICINSCHI, Holger M. zELLENTIN, (éds.), Heresy and Identity in Late Antiquity, Tübingen, Mohr Siebeck, 2008, 407 p.

1 La recherche sur les hérésies antiques est actuellement en plein renouvellement, notamment dans le cadre de ce que S.C. Mimouni appelle le «New Paradigm» (voir son article «Les origines $\mathrm{du}$ christianisme: nouveaux paradigmes ou paradigmes paradoxaux? Bibliographie sélectionnée et raisonnée», Revue biblique, 115, 2008, pp. 360-382) dont les tenants déplacent la frontière des questions d'orthodoxie et d'hérésie. L'ouvrage, édité par E.Iricinschi et H.M. Zellentin, se place dans la ligne du «New Paradigm», plutôt que dans celle initiée par Alain Le Boulluec (La notion d'hérésie dans la littérature grecque. II ${ }^{e}-I_{I}{ }^{e}$ siècles, I-II, Paris, Études augustiniennes, 1985 et «Orthodoxie et hérésie aux premiers siècles dans l'historiographie récente», dans S. Elm, E. Rebillard, A. Romano, (éds.), Orthodoxie, christianisme, histoire, Rome, 2000, pp.303-319). Résultant d'un colloque tenu, en janvier 2008, à Princeton, il se propose de revisiter la catégorie de l'hérésie, les discours sur l'hérésie et sur les hérétiques en les associant à la question des identités religieuses et en déplaçant les limites entre orthodoxies et hérésies, tant dans le christianisme que dans le judaïsme à la fin de l'Antiquité. Le fil conducteur est la manière dont l'identité de l'autre, de l'ennemi interne, est représentée et construite et dont la catégorie de l'hérésie intervient dans la formation de cette identité. Cette catégorie est en particulier appréhendée comme un label dont la fonction doit être étudiée en relation avec les desseins de ceux qui l'utilisent. 
2 Les deux éditeurs introduisent l'ensemble par quelques pages historiographiques (sur la notion d'hérésie et sur celle d'identité) et programmatiques. Deux index, des auteurs modernes et des sujets, complètent l'ensemble. Les trois premières contributions s'intéressent à l'application des catégories d'hérésie et d'orthodoxie et à leurs effets sociaux, théologiques et politiques. K.King pose la question des intentions et des stratégies des auteurs, stratégies dont elle donne une liste. Elle applique ensuite cette démarche à deux ouvrages anciens bien différents, l'un hérésiologique, l'Adversus haereses d'Irénée de Lyon, l'autre qualifié d'hérétique, l'Apocryphon de Jean, afin de voir quels sont les effets sociaux et théologiques du discours hérésiologique. W.E. Arnal considère qu'en adoptant une perspective sociologique il est possible d'utiliser le terme hérésie pour le premier siècle, même si ce terme n'est pas toujours employé; il prend ainsi le contre-pied de nombreuses recherches. Il argumente cette position en étudiant les communautés pauliniennes à partir du terme doxa tel qu'il est défini par Pierre Bourdieu (la doxa est ce qui ne fait pas l'objet de discussion). L'hérésie ne serait, alors, pas obligatoirement concomitante de l'orthodoxie et provoquerait l'opposition. Il étudie ainsi comment Paul met en place une identité qui interagit avec les identités du monde gréco-romain et comment il serait un hétérodoxe par rapport aux conceptions romaines de l'ethnicité. Cette approche mériterait une étude plus approfondie. A.Cameron s'intéresse également aux premiers chrétiens, mais son point de vue se déplace du côté de l'orthodoxie autour de deux questions: comment celle-ci se construit et comment elle génère de la violence en menant une politique coercitive.

3 Les contributions suivantes reviennent sur certains de ces points à partir de cas plus précis. Comme A.Cameron, Y. Papadoyannakis se penche sur la construction de l'orthodoxie, en montrant qu'il s'agit d'un processus long. L'étude de la stratégie littéraire du pseudo-Justin dans Quaestiones et responsiones ad orthodoxos témoigne de la manière dont l'orthodoxie se construit à travers les solutions apportées à des problèmes posés par les «autres». C.Humfress livre une étude intéressante de la législation impériale post-constantinienne relative à la citoyenneté. Tout en se fondant dans un cadre ancien, les empereurs chrétiens ont innové, comme en témoigne l'évolution du champ d'application du concept d'infamie qui est étendu à l'hérésie et l'apostasie. R.Lim revient, quant à lui, sur la question du label. Son article se situe dans la ligne de ce que K.King (What is Gnosticism, Cambridge-London, Belknap Press, 2003) et M.A. Williams (Rethinking «Gnosticism». An Argument for Dismantling a Dubious Category, Princeton, Princeton University Press, 1996) ont fait pour les gnostiques: remettre en question la pertinence d'une telle catégorie, en s'interrogeant sur les raisons de l'emploi du label «manichéen». Il considère que son emploi comme auto-identité est plus une exception qu'une norme et que ce terme désigne surtout l'autre. On peut reprocher à R.Lim de ne pas prendre en compte les sources directes de Kellis, notamment les lettres retrouvées en ce lieu. De plus, comme cela a déjà été soulevé pour les gnostiques, si ce concept n'est peut-être pas le plus adéquat, il n'en reste pas moins que ceux qui sont qualifiés de manichéens, tout en étant chrétiens, se distinguent des autres chrétiens par certaines caractéristiques incontestables et qu'il faut bien un terme pour les distinguer.

4 Le thème des articles suivants concerne la question de l'identité des juifs et des premiers chrétiens. K.E. Esterloh étudie l'influence de Rome et de la romanité sur l'identité juive, tandis que P.Townsend s'interroge de manière convaincante sur les référents possibles au terme christianoi, dont le sens normatif n'apparaitrait qu'avec 
Ignace, au moment où la distinction avec le judaïsme s'accroît. J.G. Gager, dans un court article où il adopte explicitement un point de vue nominaliste, porte son intérêt sur l'anti-judaïsme lucanien. Selon lui, il s'agirait d'un moyen de stigmatiser des groupes semblables à celui auquel l'auteur appartient, ennemis internes ainsi décrits comme des ennemis externes. Cette thématique des ennemis internes se retrouve aussi dans l'article d'E. Pagels sur Ignace d'Antioche et l'Apocalypse de Jean. Elle se concentre surtout sur la thématique du mal et son exploitation par l'auteur de l'Apocalypse.

Les deux articles suivants déplacent la frontière entre hérésie et orthodoxie en ne considérant pas les textes étudiés comme hérétiques, comme c'est habituellement le cas dans la recherche. Le premier, E. Iricinschi, pour l'Évangile de Philippe, considère, à juste titre, que cela fausserait l'analyse, le second, A.Y. Reed, que les homélies pseudoclémentines participent aussi du discours hérésiologique avec les mêmes méthodes. E.Iricinschi se concentre sur le rôle du label «hébreux» pour caractériser d'autres groupes pauliniens concurrents, sur l'usage des métaphores familiales et sur le recours à un langage différent pour marquer la différence entre groupes concurrents.

6 Les derniers articles du volume portent sur la construction de l'autre dans la littérature rabbinique. B.L. Visotzky étudie la notion rabbinique d'hérésie dans la polémique contre les Gentils, tandis que G.Gardner s'intéresse à la manière dont le Talmud stigmatise les astrologues afin d'en faire des marqueurs distinctifs entre Israël et les Gentils. H.M. Zellentin part également du Talmud, de l'histoire d'Imma Shalom et du philosophe, pour montrer la manière dont le Talmud polémique contre le christianisme en le ridiculisant. I.J.Yuval utilise un autre corpus, celui des prières: les confronter à la liturgie chrétienne permettrait d'y relever des traits polémiques (polémique qui n'est cependant pas directe) et de comprendre certaines de leurs modifications faites en fonction du christianisme.

7 Même si certains arguments peuvent ne pas être toujours convaincants, l'ensemble des contributions est stimulant pour la recherche; en effet, l'ensemble propose de nouveaux usages heuristiques de la catégorie d'hérésie, déplace les lignes de démarcation entre hérésie et orthodoxie et suggère de nouvelles approches pour les textes. Dans le même temps, il s'agit d'une nouvelle invitation à faire attention à l'utilisation de ces catégories et à ne pas se laisser enfermer par elles. 